\title{
The MicroTrak test for rapid detection of chlamydiae in diagnosing and managing women with abdominal pain
}

\author{
P E MUNDAY, B J THOMAS, AND D TAYLOR-ROBINSON \\ From the Division of Sexually Transmitted Diseases, MRC Clinical Research Centre, Harrow, Middlesex, and the \\ Praed Street Clinic, St Mary's Hospital, London
}

SUMMARY Using a fluorescein labelled monoclonal antibody ("MicroTrak") to identify chlamydia elementary bodies in endocervical smears, we detected Chlamydia trachomatis in 31 (21\%) of 150 case of classic pelvic inflammatory disease (PID) and in $42(18 \%)$ of 232 cases of abdominal pain not diagnosed as PID. Only $43(59 \%)$ of the women yielding chlamydiae would have received treatment in the absence of a diagnostic service for chlamydial infection. Evidence of chlamydial infection should be sought in all women presenting to a sexually transmitted disease (STD) clinic with abdominal pain.

\section{Introduction}

Chlamydia trachomatis is recognised as an important cause of pelvic inflammatory disease (PID). ${ }^{1}$ The results of studies conducted in Sweden suggest that women with chlamydial PID often have symptoms and signs that are mild compared with the severity of the pathological changes observed in these women at laparoscopy. ${ }^{2}$ Without laparoscopy, not only is it difficult for the clinician to assess the severity of the disease but also the clinical diagnosis of PID itself is subject to considerable error. Thus Jacobson and Westrom found that of women who underwent laparoscopy after a diagnosis of PID had been made by an experienced gynaecologist, only $65 \%$ had visual evidence of the disease. ${ }^{3}$ The fact that the clinical diagnosis of PID by experienced gynaecologists is subject to so large a degree of error raises anxiety about the management of women attending sexually transmitted disease (STD) clinics with abdominal pain and in whom a diagnosis of PID is considered. Thus clinicians may overdiagnose and overtreat to avoid the organic sequelae of the disease, only to leave healthy women with the psychological burden of presumed PID. On the other hand, some doctors may believe that treatment should be avoided unless there is evidence of "classic PID", thus leaving untreated women with mild disease. If the contention of Svensson et al, ${ }^{2}$ mentioned above, is valid widely, Address for reprints: Dr P E Munday, Praed Street Clinic, St Mary's
Hospital, Paddington, London W2 IPG

Accepted for publication 14 August 1985 many of such untreated women are likely to have a chlamydial infection.

The opportunity to test this hypothesis arose at the Praed Street STD Clinic in May 1984 with the introduction of a limited chlamydial diagnostic service based on the MicroTrak test. We present here an analysis of the results of tests on specimens obtained from women complaining of abdominal pain during the year to 30 April 1985.

\section{Patients and methods}

We offered a chlamydial diagnostic service for all women attending the Praed Street Clinic who complained of abdominal pain. Doctors were asked not to submit specimens from patients who had been treated with antibiotics in the previous four weeks unless reinfection was suspected. A specimen was taken from the endocervix with a polyester sponge $\mathrm{swab}^{4}$ or a cotton tipped swab, and was wiped over the unmasked area of a slide. The material was air dried, fixed in acetone, and stored at $4^{\circ} \mathrm{C}$ until the following morning. The slides were then allowed to reach room temperature and the fixed material was stained with a monoclonal antibody linked to fluorescein ("MicroTrak", Syva, England) and examined for chlamydial elementary bodies using a fluorescence microscope. The detection of one or more elementary bodies was considered to be evidence of chlamydial infection. ${ }^{5}$

We scrutinised the case notes of all women from whom a specimen had been submitted with a request form mentioning abdominal pain or PID. Patients were excluded from the analysis if they had taken 
antibiotics in the previous four weeks or if the diagnosis of PID had been made on the basis of a symptom other than abdominal pain, such as deep dyspareunia, or on physical signs in the absence of pain. Patients were also excluded if the pain could be attributed to another condition, such as a urinary tract infection, for which the diagnosis was reasonably certain at the time the cervical specimen was obtained.

Details were obtained of the patient's contact history, symptoms, signs, clinical diagnosis, and the presence or absence of infection with Neisseria gonorrhoeae. In cases where no diagnosis was stated, we assumed that a diagnosis of PID was intended if the doctor had chosen to prescribe the clinic's standard treatment regimen for PID.

\section{Results}

During the 12 months, 489 specimens were submitted for chlamydial tests allegedly because of abdominal pain or PID. Analysis of case records disclosed that 24 were from patients who had recently taken antibiotics, 65 from women without abdominal pain, nine from women with other clinical diagnoses, and nine specimens were inadequate for obtaining a valid result. $C$ trachomatis elementary bodies were identified in $73(19 \%)$ of the remaining 382 specimens. In 150 cases the examining doctor had diagnosed PID and $31(21 \%)$ of the specimens were chlamydia positive. A diagnosis of abdominal pain not associated with PID was made in the other cases and $42(18 \%)$ were chlamydia positive, a difference that was not significant $(\mathrm{p}>0.05)$.

$N$ gonorrhoeae was isolated from 37 women, 16 of whom were diagnosed as having PID and 21 of whom were thought to have non-PID abdominal pain. Among the women yielding $N$ gonorrhoeae, $C$ trachomatis was identified in specimens from six (38\%) of those with PID and from nine (43\%) of those with non-PID abdominal pain. An additional 10 women were sexual contacts of men with gonorrhoea or nongonococcal urethritis, and $C$ trachomatis was detected in three of them.

\section{Discussion}

The results of this study indicate that women attending an STD clinic who have abdominal pain considered not to be associated with PID are as likely as those who have "classic PID" to have chlamydial infection of the cervix. It would be wrong, of course, to assume that either group of women had upper genital tract infection due to chlamydiae, as it has been shown that cervical infection does not necessarily predict which micro-organisms are simultaneously present in the fallopian tubes. ${ }^{6}$ Nevertheless, chlamydiae may cause PID, and if the micro-organisms are isolated from the cervix of a woman who has signs of, but no evidence of another cause of, PID it would be wrong not to manage her as though she had a chlamydial salpingitis. It might be argued similarly, particularly in view of the known limitations of clinical examination, that any woman with chlamydial infection of the cervix and lower abdominal pain with no other cause of pain should be regarded as having chlamydial PID. This policy would undoubtedly lead to overdiagnosis of PID, which could be resolved only by undertaking laparoscopy of all women with lower genital tract infection and lower abdominal pain, however mild.

Our observations indicate also that lower abdominal pain should be added to the list of indications for diagnosis of chlamydial infection when access to a diagnostic service is limited. It was interesting that only a small proportion of patients came from known risk groups for chlamydial infection. "Blind" antichlamydial treatment is our clinic policy for patients with PID and gonorrhoea and for those who present as sexual contacts of men with nongonococcal urethritis. Such patients comprised 43 $(59 \%)$ of the 73 chlamydia positive patients studied. In the absence of the diagnostic service, therefore, about $40 \%$ of chlamydia positive patients would have been untreated. The consequences of such a failure are unknown but many such women would possibly need investigation and treatment for tubal infertility in the future, unaware of ever having had a genital tract infection.

We thank the medical, nursing and reception staff of the Praed Street Clinic for their cooperation and Drs JRW Harris and D Goldmeier for access to data relating to patients under their care.

\section{References}

1. Weström L, Mårdh P-A. Chlamydial salpingitis. Br Med Bull 1983;39:145-50.

2. Svensson L, Westöm L, Ripa KT, Mårdh P-A. Differences in some clinical and laboratory parameters in acute salpingitis related to culture and serologic findings. Am J Obstet Gynecol 1980;138:1017-21.

3. Jacobson L, Westöm L. Objectivized diagnosis of acute pelvic inflammatory disease: diagnostic and prognostic value of routine laparoscopy. Am J Obstet Gynecol 1969;105:1088-98.

4. Oates JK, Selwyn S, Breach MB. Polyester-sponge swabs to facilitate examination for genital infection in women. British Journal Venereal Diseases 1971;47:289-92.

5. Thomas BJ, Evans RT, Hawkins DA, Taylor-Robinson D. Sensitivity of detecting Chlamydia trachomatis elementary bodies in smears by use of a fluorescein labelled monoclonal anitbody: comparison with conventional chlamydial isolation. J Clin Pathol 1984;37:812-6.

6. Sweet RL, Schachter J, Robbie MO. Failure of B lactam antibiotics to eradicate Chlamydia trachomatis in the endometrium despite apparent clinical cure of acute salpingitis. JAMA 1984;250:2641-5. 